\title{
Vulnerabilidades clínicas e sociais em usuários de crack de acordo com a situação de moradia: um estudo multicêntrico em seis capitais brasileiras
}

\section{Clinical and social vulnerabilities in crack users according to housing status: a multicenter study in six Brazilian state capitals}

\section{Vulnerabilidades clínicas y sociales en consumidores de crack, de acuerdo con el acceso a la vivienda: un estudio multicéntrico en seis capitales brasileñas}

Silvia Chwartzmann Halpern

Juliana Nichterwitz Scherer 1

Vinicius Roglio 1

Sibele Faller 1

Anne Sordi 1

Felipe Ornell 1

Carla Dalbosco 1

Flavio Pechansky 1

Félix Kessler 1

Lisia von Diemen 1

\section{Resumo}

O estudo teve como objetivo avaliar a gravidade do uso de substâncias psicoativas, situações de violência, saúde física e emocional de usuários de crack que buscam atendimento em Centros de Atenção Psicossocial para Álcool e Drogas (CAPSad), em relação ao status de moradia. Trata-se de um estudo multicêntrico em seis capitais brasileiras, com 564 usuários de crack categorizados em dois grupos: (1) usuários que estiveram em situação de rua $(n=266) e$ (2) nunca estiveram em situação de rua $(n=298)$. Para avaliar a gravidade do uso de substâncias e as características dos indivíduos utilizou-se o Addiction Severity Index, 6a versão (ASI-6). Os usuários do grupo 1 demonstraram piores indicadores em relação às subescalas álcool, problemas médicos, psiquiátricos, trabalho e suporte familiar, além de maior envolvimento com problemas legais, violência, abuso sexual, risco de suicídio e problemas de saúde como HIV/AIDS, hepatite e tuberculose, além de possuirem menos renda para pagar necessidades básicas. Após análises multivariadas ajustadas para possiveis confundidores, não possuir renda suficiente para pagar necessidades básicas, apresentar sintomas depressivos e ter sido preso por roubo permaneceram significativos. Este trabalho avaliou de forma mais abrangente a gravidade do uso de drogas e a situação de moradia de usuários de crack. Abordagens desenvolvidas nos dispositivos de tratamento extra-hospitalares devem ser projetadas e adequadas de acordo com perfis e demandas especificos dos usuários, em especial aqueles em situação de rua.

Transtornos Relacionados ao Uso de Substâncias; Crack; Pessoas em Situação de Rua; Vulnerabilidade em Saúde; Vulnerabilidade Social

\author{
Correspondência \\ S. C. Halpern \\ Centro de Pesquisa em Álcool e Drogas, Hospital de Clínicas de \\ Porto Alegre, Universidade Federal do Rio Grande do Sul. \\ Rua Professor Álvaro Alvim 400, Porto Alegre, \\ RS 90420-020, Brasil. \\ silvia.halpern@gmail.com \\ 1 Hospital de Clínicas de Porto Alegre, Universidade Federal do \\ Rio Grande do Sul, Porto Alegre, Brasil.
}




\section{Introdução}

O abuso de crack é considerado um problema de saúde pública e está associado à violência e criminalidade, a problemas psicológicos, sociais, ocupacionais e à potencialização da contaminação por doenças infectocontagiosas 1 . Estima-se que a prevalência do uso regular de crack ou similares nas capitais brasileiras seja de 0,81\%, correspondendo a 35\% dos consumidores de drogas ilícitas, excluindo a maconha 2. Estudos apontam para um aumento significativo no consumo de crack no Brasil nos últimos anos, possivelmente em razão de mudanças referentes a novas formas de uso de cocaína, ao acesso e a estratégias de mercado 3,4. Apesar das taxas de consumo do crack serem inferiores a de outras drogas, observa-se que esta é a droga ilícita que mais conduz a internações em hospitais psiquiátricos e a que mais provoca demanda por atendimento, gerando um custo expressivo para o sistema público de saúde 5,6,7.

Os usuários de crack caracterizam-se por serem uma população de risco e apresentarem inúmeras vulnerabilidades, tornando-se um desafio para os serviços de tratamento e para as políticas públicas da área da saúde e da assistência social. A exposição a doenças e a situações adversas da vida acontece de forma diferenciada de acordo com cada indivíduo, regiões e grupos sociais, e ela está intimamente relacionada às condições socioeconômicas, ao nível educacional e a outros indicadores sociais, como status de moradia, situação legal e doenças associadas $2,8,9$.

Muito embora não exista uma definição única para a conceituação de indivíduos em situação de rua, o viver na rua implica uma condição em que há inexistência de moradia estável 10, utilização de áreas públicas e degradadas como espaço de moradia e sustento, existência de vínculos familiares rompidos e vida em situação de pobreza extrema 11,12. Nesse contexto, a literatura demonstra uma importante interface entre o uso de substâncias e essa população em especial.

Em especial, a condição de morar na rua está associada a uma série de vulnerabilidades de saúde, sociais e legais, que exacerbam sua marginalização social. Especificamente, quando privados de acesso a serviços e ações de prevenção e apoio social, os usuários estão em maior risco de sofrerem abuso sexual, apresentarem algum diagnóstico de transtorno mental, bem como múltiplas comorbidades clínicas 10,13. Entretanto, observa-se uma insuficiência de informações a respeito de como a vulnerabilidade social presente em usuários de crack, em especial naqueles indivíduos em situação de rua, pode estar relacionada à gravidade de uso de determinadas substâncias psicoativas. Nesse sentido, o presente estudo tem como objetivo avaliar a gravidade do uso de substâncias psicoativas, situações de violência, saúde física e emocional de usuários de crack que buscam atendimento em Centros de Atenção Psicossocial para Álcool e Drogas (CAPSad), em relação ao status de moradia.

\section{Material e métodos}

\section{Desenho do estudo e amostragem}

Este trabalho é uma análise de dados secundários a um estudo transversal multicêntrico que teve como objetivo principal a avaliação, gerenciamento de caso e o seguimento de usuários de crack em tratamento em seis capitais brasileiras: Brasília (Distrito Federal), Porto Alegre (Rio Grande do Sul), Rio de Janeiro, Salvador (Bahia), São Paulo e Vitória (Espírito Santo) 14. O estudo principal realizou o recrutamento dos participantes da pesquisa valendo-se de uma amostragem consecutiva de conveniência. O cálculo amostral foi baseado nos desfechos previstos, sendo feito um acréscimo de $20 \%$ para compensar possíveis perdas no seguimento. O desfecho "morador de rua" não influenciou originalmente o cálculo amostral. Dessa forma, foi identificado um total de 765 usuários de crack que buscavam tratamento na rede pública.

A coleta de dados foi realizada entre abril de 2011 e dezembro de 2012 por meio de entrevistas realizadas na primeira semana após o ingresso no tratamento (internação ou CAPSad). Os critérios de inclusão utilizados para a composição da amostra do estudo principal foram: usar crack como droga de preferência, ser maior de 18 anos, estar em tratamento para dependência química em algum dos locais de recrutamento citados antes, e preencher os critérios para dependência de cocaína segundo o DSM-IV-TR (Manual Diagnóstico e Estatístico de Transtornos Mentais) 15. Os sujeitos que não comple- 
taram a avaliação inicial, assim como aqueles que possuíam algum impedimento emocional ou físico que limitasse o preenchimento dos instrumentos foram excluídos do estudo. As equipes de coleta foram treinadas sob a responsabilidade dos coordenadores regionais dos seis centros de pesquisa, contando com o auxílio técnico e a supervisão presencial dos profissionais do Centro de Pesquisa em Álcool e Drogas (CPAD) do Hospital de Clínicas de Porto Alegre, Universidade Federal do Rio Grande do Sul (HCPA/UFRGS). Foram envolvidos seis coordenadores regionais e 24 coletadores nos diferentes estados, que também revisaram e controlaram a qualidade das coletas e a inserção dos dados.

No presente trabalho foram usados somente os dados referentes aos usuários que buscaram atendimento nos CAPSad. Após o tratamento dos dados com a exclusão daqueles que não finalizaram a avaliação inicial e conforme os critérios de inclusão deste estudo, totalizou-se uma amostra final de 564 usuários de crack.

\section{Instrumentos e variáveis de análise}

Para avaliar o impacto do uso de substâncias, foi utilizado o Addiction Severity Index, 6ạ versão (ASI-6), que consiste em entrevista semiestruturada multidimensional que avalia o impacto do uso de substâncias na vida do paciente em sete áreas de funcionamento: médico, trabalho, aspectos legais, aspectos sociofamiliares, psiquiátrico e uso de álcool e outras drogas. A utilização e validação do instrumento podem ser encontradas em publicações anteriores 16,17 . O status de moradia foi avaliado pela questão H10: "Alguma vez na vida você já ficou em um abrigo para moradores de rua ou na rua (em lugares como prédios abandonados, carros, parques ou praças) porque você não tinha outro lugar para ficar?”. Os sujeitos que responderam sim a essa questão foram incluídos no grupo "morou na rua" ( $\mathrm{n}=266)$, e os que responderam não foram incluídos no grupo "não morou na rua" ( $\mathrm{n}=298)$.

\section{Análise estatística}

Os dados coletados foram analisados no IBM SPSS (IBM Corp, Armonk, Estados Unidos), versão 18.0. A descrição dos dados categóricos foi realizada pela frequência absoluta e relativa em tabelas de contingência, e associações foram investigadas pelo teste qui-quadrado de Pearson. As variáveis com valor de $\mathrm{p}<0,10$ nas análises bivariadas foram selecionadas para inclusão no modelo multivariado. Quando havia mais de uma variável avaliando o mesmo constructo teórico, foi escolhida aquela com associação mais forte com o desfecho. Em seguida, utilizou-se o modelo de regressão de Poisson múltipla para estimar razão de prevalência. A fim de testar a multicolinearidade do modelo foi calculado o fator de inflação de variância (VIF) 18. A normalidade das distribuições das variáveis quantitativas foi analisada pelo teste Kolmogorov Smirnov e apresentada por média e desvio padrão (DP). Os subescores do ASI- 6 foram comparados pelo teste $t$ de Student para amostras independentes quando comparados dois grupos com nível de $5 \%$ de significância.

\section{Aspectos éticos}

O presente projeto ocorreu em acordo com as normas éticas e foi aprovado pela Comissão de Ética em Pesquisa do HCPA/UFRGS número 07/2014: \#14-0395, CAAE 31140014200005327. O projeto principal e os Termos de Consentimento Livre e Esclarecidos (TCLEs) foram replicados e submetidos aos comitês locais dos centros participantes e aprovados sob o número 100176. Os indivíduos avaliados que concordaram em participar do estudo preencheram o TCLE.

\section{Resultados}

Dos 564 indivíduos avaliados em todos os estados, 266 (47,1\%) estiveram em situação de rua em algum momento da vida e 298 (42,9\%) nunca viveram na rua. Além disso, verificou-se que 69,3\% e 37,8\% dos sujeitos que relataram ter morado na rua durante a vida estiveram em situação de rua pelo menos uma vez nos últimos seis meses e 30 dias, respectivamente. Dentre os resultados encontrados, a situação de moradia foi um marcador de vulnerabilidade social e é apresentada na Tabela 1. 
Tabela 1

Características sociodemográficas de acordo com a situação de moradia na rua de usuários de crack de seis capitais brasileiras.

\begin{tabular}{|c|c|c|c|c|}
\hline & $\begin{array}{c}\text { Morou na rua } \\
{[n=266(47,2 \%)]}\end{array}$ & $\begin{array}{c}\text { Nunca morou na rua } \\
{[n=298(52,8 \%)]}\end{array}$ & $\begin{array}{c}\text { Total } \\
{[N=564(100,0 \%)]}\end{array}$ & Valor de $p$ \\
\hline Idade * & $32,4 \pm 8,7$ & $32,6 \pm 8,5$ & $32,5 \pm 8,6$ & 0,829 \\
\hline \multicolumn{5}{|l|}{ Gênero ** } \\
\hline Masculino & $216(81,5)$ & $240(80,8)$ & $456(81,1)$ & \multirow{2}{*}{0,914} \\
\hline Feminino & $49(18,5)$ & $57(19,2)$ & $106(18,9)$ & \\
\hline \multicolumn{5}{|l|}{ Raça ** } \\
\hline Negra & $86(32,3)$ & $91(30,5)$ & $177(31,8)$ & \multirow{4}{*}{0,880} \\
\hline Branca & $79(29,7)$ & $96(32,2)$ & $175(31,0)$ & \\
\hline Parda/Mestiça & $92(34,6)$ & $90(30,2)$ & $182(32,3)$ & \\
\hline Outra & $9(3,4)$ & $21(7,0)$ & $30(5,3)$ & \\
\hline \multicolumn{5}{|l|}{ Estado civil ** } \\
\hline Casado ou vivendo como casado & $71(26,8)$ & $101(34,1)$ & $172(30,7)$ & \multirow[b]{3}{*}{0,660} \\
\hline Separado, divorciado ou viúvo & $91(34,3)$ & $78(26,4)$ & $169(30,1)$ & \\
\hline Nunca casou & $103(38,9)$ & $117(39,5)$ & $220(39,2)$ & \\
\hline \multicolumn{5}{|l|}{ Escolaridade ** } \\
\hline Nenhuma & $38(14,3)$ & $30(10,1)$ & $68(12,1)$ & \multirow{3}{*}{0,094} \\
\hline Ensino Fundamental & $134(50,4)$ & $139(46,6)$ & $273(48,4)$ & \\
\hline Ensino Médio ou mais & $94(35,3)$ & $129(43,3)$ & $223(39,5)$ & \\
\hline \multicolumn{5}{|l|}{ Renda últimos 6 meses (SM) ** } \\
\hline Sem renda & $72(27,6)$ & $79(27,2)$ & $151(27,4)$ & \multirow{5}{*}{0,747} \\
\hline Menos de 1 & $27(10,3)$ & $27(9,3)$ & $54(9,8)$ & \\
\hline $1-3$ & $40(15,3)$ & $37(12,8)$ & $77(14,0)$ & \\
\hline $3-5$ & $17(6,5)$ & $26(9,0)$ & $43(7,8)$ & \\
\hline Mais de 5 & $105(40,2)$ & $121(41,7)$ & $226(41,0)$ & \\
\hline Não possui renda suficiente para pagar necessidades básicas ** & $199(74,8)$ & $182(61,1)$ & $381(67,6)$ & 0,001 \\
\hline \multicolumn{5}{|l|}{ Emprego ** } \\
\hline Trabalho formal/Trabalho informal & $48(18,0)$ & $76(25,6)$ & $124(22,0)$ & \multirow{2}{*}{0,031} \\
\hline Desempregado/Fora do trabalho & $218(82,0)$ & $221(74,4)$ & $439(78,0)$ & \\
\hline
\end{tabular}

SM: salário mínimo.

* Representação por média \pm desvio padrão; distribuição normal; teste t para amostras independentes;

** Representação por $n$ e percentual; teste de qui-quadrado de associação.

Nota: salário mínimo à época do estudo no valor de $\mathrm{R} \$ 545,00$. Os valores de " $\mathrm{n}$ ” podem apresentar diferenças devido aos missings de cada variável.

Ao serem analisadas as diferentes dimensões do ASI-6, percebe-se que os indivíduos que, em algum momento estiveram em situação de rua ou em abrigos (grupo 1), mostraram médias mais elevadas e estatisticamente significativas em relação às subescalas álcool, problemas médicos e psiquiátricos. Da mesma forma, embora com menor diferença, mas ainda estatisticamente significativa, esses indivíduos apresentaram mais problemas nas subescalas trabalho e suporte social e familiar em comparação com os indivíduos do grupo 2 (Tabela 2).

Os resultados também apontam para uma associação significativa entre alguns aspectos da saúde física, emocional, envolvimento legal e violência com a situação de moradia (Tabelas 3 e 4). A condição de ter morado na rua em algum momento da vida esteve associada a ter sido preso ou detido por porte de drogas $(28,8 \%$ vs. $20,8 \%$; $\mathrm{p}=0,031)$, por roubo $(30,3 \% v s .16,7 \%$; $\mathrm{p}<0,001)$ e por crime violento (11\% vs. 5,1\%; $\mathrm{p}=0,012$ ). Da mesma forma, essa condição apresentou associação com ter sofrido agressão física $(55,5 \%$ vs. $45,1 \% ; \mathrm{p}=0,018)$, agressão ou abuso sexual $(18,1 \%$ vs. $11,1 \% ; \mathrm{p}=0,022)$ e ter sido vítima de crime violento $(52,5 \%$ vs. $38,9 \%$; $\mathrm{p}=0,001)$. Por fim, no que diz respeito à saúde física 
Tabela 2

Pontuações na Addiction Severity Index, 6a versão (ASI-6) por status de moradia de usuários de crack de seis capitais brasileiras.

\begin{tabular}{lccc}
\hline Subescalas do ASI-6 & $\begin{array}{c}\text { Morou na rua } \\
\mathbf{( n = 2 6 6 )}\end{array}$ & $\begin{array}{c}\text { Não morou na rua } \\
(\mathbf{n}=\mathbf{2 9 8})\end{array}$ & $\begin{array}{l}\text { Valor de } \mathbf{p} \\
\text { Drogas }\end{array}$ \\
Álcool & $50,9 \pm 7,3$ & $50,5 \pm 6,8$ & 0,357 \\
Psiquiátrica & $50,7 \pm 9,9$ & $48,3 \pm 9,1$ & $\mathbf{0 , 0 0 3}$ \\
Médica & $51,2 \pm 8,5$ & $48,4 \pm 8,6$ & $\mathbf{0 , 0 0 0}$ \\
Legal & $50,8 \pm 9,9$ & $46,7 \pm 8,6$ & $\mathbf{0 , 0 0 0}$ \\
Emprego/Sustento & $50,7 \pm 6,9$ & $49,7 \pm 6,1$ & 0,100 \\
Apoio social e familiar & $39,2 \pm 4,7$ & $37,9 \pm 5,9$ & $\mathbf{0 , 0 2 8}$ \\
Problemas familiares & $38,4 \pm 8,6$ & $36,4 \pm 7,5$ & $\mathbf{0 , 0 0 7}$ \\
\hline
\end{tabular}

Nota: representação por média \pm desvio padrão; teste de Mann-Witney. Em negrito valores de $p$ significativos ( $p<0,05)$.

\section{Tabela 3}

Comparação da violência entre usuários de crack em tratamento em Centros de Atenção Psicossocial para Álcool e Drogas (CAPSad) de seis capitais brasileiras de acordo com a situação de moradia.

\begin{tabular}{|c|c|c|c|c|}
\hline & $\begin{array}{l}\text { Morou na rua } \\
\text { (n= 266) } \\
n(\%)\end{array}$ & $\begin{array}{c}\text { Nunca morou na rua } \\
\qquad(\mathrm{n}=298) \\
n(\%)\end{array}$ & $\begin{array}{c}\text { Total } \\
(\mathrm{N}=564) \\
\mathrm{n}(\%)\end{array}$ & Valor de p \\
\hline Já esteve preso ou detido & $191(72,1)$ & $170(57,2)$ & $361(64,2)$ & $<0,001$ \\
\hline Preso antes dos 18 anos & $29(11,2)$ & $24(8,2)$ & $53(9,6)$ & 0,252 \\
\hline Preso por porte de drogas & $76(28,8)$ & $61(20,8)$ & $137(24,6)$ & 0,031 \\
\hline Preso por venda e produção de drogas & $22(8,3)$ & $21(7,2)$ & $43(7,7)$ & 0,363 \\
\hline Preso por roubo & $80(30,3)$ & $49(16,7)$ & $129(23,2)$ & $<0,001$ \\
\hline Preso por crime violento & $29(11,0)$ & $15(5,1)$ & $44(7,9)$ & 0,012 \\
\hline Fisicamente agredido/abusado por alguém que conhecia & $147(55,5)$ & $134(45,1)$ & $281(50,0)$ & 0,018 \\
\hline Agredido/Abusado sexualmente & $48(18,1)$ & $33(11,1)$ & $81(14,4)$ & 0,022 \\
\hline Vítima de um crime violento (p. ex.: espancado/agredido) & $139(52,5)$ & $115(38,9)$ & $254(45,3)$ & 0,001 \\
\hline
\end{tabular}

Nota: teste de qui-quadrado exato de Fisher. Em negrito valores de $p$ significativos $(p<0,05)$. Os valores de " $n$ " podem apresentar diferenças devido aos missings de cada variável.

e emocional do grupo em situação de rua, o item apresentou associação com a presença de infecção por HIV (6,8\% vs. $2 \% ; \mathrm{p}=0,006)$, tuberculose $(8,3 \%$ vs. $2,7 \% ; \mathrm{p}=0,004)$, hepatite $(12 \%$ vs. $6,8 \% ; \mathrm{p}=$ $0,041)$, internamentos por problemas psicológicos e psiquiátricos $(15,8 \% v s .9,7 \% ; \mathrm{p}=0,031)$, sintomas depressivos $(58,1 \%$ vs. $47,6 \%$; $\mathrm{p}<0,001)$ e de ideação suicida $(49,4 \%$ vs. $37,4 \%$; $\mathrm{p}=0,002)$.

Entre os sujeitos que afirmaram ter morado na rua, identificou-se que a mediana (intervalo interquartílico) de dias morados na rua foi maior em indivíduos que foram vítimas de crimes violentos [30 (7-90) vs. 12 (3,5-30); $\mathrm{p}=0,008$ ] e agredidos fisicamente [25 (7-90) vs. 12 (3,75-48); $\mathrm{p}=0,016]$. Além disso, indivíduos que relataram ter contraído tuberculose também estiveram mais dias na rua nos últimos seis meses [50 (21-90) vs. 18,5 (4-60); $\mathrm{p}=0,027]$.

Com o objetivo de estimar a razão de prevalência (RP) entre as vulnerabilidades sociais e a situação de moradia, utilizou-se o modelo de regressão de Poisson. Os resultados estão apresentados na Tabela 5. Os VIFs calculados apresentaram valores inferiores a 2, não evidenciando problema de 


\section{Tabela 4}

Comparação da condição de saúde física e emocional entre usuários de crack em tratamento em Centros de Atenção Psicossocial para Álcool e Drogas (CAPSad) de seis capitais brasileiras de acordo com a situação de moradia.

\begin{tabular}{|c|c|c|c|c|}
\hline & $\begin{array}{l}\text { Morou na rua } \\
(n=266) \\
n(\%)\end{array}$ & $\begin{array}{l}\text { Nunca morou na rua } \\
\qquad(\mathrm{n}=298) \\
n(\%)\end{array}$ & $\begin{array}{c}\text { Total } \\
(\mathrm{N}=564) \\
\mathrm{n}(\%)\end{array}$ & Valor de $p$ \\
\hline HIVIAIDS * & $18(6,8)$ & $6(2,0)$ & $24(4,3)$ & 0,006 \\
\hline Tuberculose * & $22(8,3)$ & $8(2,7)$ & $30(5,3)$ & 0,004 \\
\hline Hepatite * & $32(12,0)$ & $20(6,8)$ & $52(9,3)$ & 0,041 \\
\hline Cirrose ou outra doença no fígado * & $7(2,6)$ & $7(2,4)$ & $14(2,5)$ & 0,999 \\
\hline Recebeu pensão para doença física/incapacidade & $47(17,7)$ & $36(12,1)$ & $83(14,7)$ & 0,074 \\
\hline Hospitalização clínica & $172(64,7)$ & $185(62,1)$ & $357(63,3)$ & 0,541 \\
\hline Internado por problemas psicológicos/psiquiátricos & $42(15,8)$ & $29(9,8)$ & $71(12,6)$ & 0,031 \\
\hline \multicolumn{5}{|l|}{ Sintomas depressivos } \\
\hline Não & $48(18,1) * *$ & $102(34,7) * \star$ & $150(26,8)$ & \\
\hline $\operatorname{Sim}$ & $154(58,1) * *$ & $140(47,6) * *$ & $294(52,6)$ & $<0,001$ \\
\hline Somente sob efeito de droga ou em abstinência & $63(23,8)$ & $52(17,7)$ & $115(20,6)$ & \\
\hline \multicolumn{5}{|c|}{ Dificuldade para controlar temperamento e impulsos violentos } \\
\hline Não & $145(54,7)$ & $181(60,9)$ & $326(58,0)$ & \\
\hline Sim & $91(34,3)$ & $82(27,6)$ & $173(30,8)$ & 0,220 \\
\hline Somente sob efeito de droga ou em abstinência & $29(10,9)$ & $34(11,4)$ & $63(11,2)$ & \\
\hline \multicolumn{5}{|l|}{ Ideação suicida } \\
\hline Não & $95(35,8) * *$ & $150(50,5)^{\star}$ & $245(43,6)$ & \\
\hline $\operatorname{Sim}$ & $131(49,4) * *$ & $111(37,4)^{*}$ & $242(43,1)$ & 0,002 \\
\hline Somente sob efeito de droga ou em abstinência & $39(14,7)$ & $36(12,1)$ & $75(13,3)$ & \\
\hline \multicolumn{5}{|l|}{ Tentou o suicídio } \\
\hline Não & $164(61,9)$ & $210(70,7)$ & $374(66,5)$ & \\
\hline $\operatorname{Sim}$ & $75(28,3)$ & $64(21,5)$ & $139(24,7)$ & 0,086 \\
\hline Somente sob efeito de droga ou em abstinência & $26(9,8)$ & $23(7,7)$ & $49(8,7)$ & \\
\hline
\end{tabular}

* Autorrelato;

** Resíduo ajustado > $|2|$.

Nota: teste de qui-quadrado. Em negrito valores de $p$ significativos $(p<0,05)$. Os valores de " $n$ " podem apresentar diferenças devido aos missings de cada variável.

multicolinearidade no modelo. Dentro do grupo de variáveis preditoras, foi mais prevalente nos moradores de rua ter sido preso por roubo $(R P=1,32 ; \mathrm{p}=0,002)$ e apresentar sintomas depressivos $(\mathrm{RP}=1,512 ; \mathrm{p}=0,002)$.

\section{Discussão}

Este estudo de caráter multicêntrico avaliou de forma mais abrangente a gravidade do uso de drogas e situação de moradia de usuários de crack em tratamento em CAPSad no Brasil. Usuários nas diferentes regiões do país apresentaram escores significativos de gravidade de uso, demonstrando que ter um histórico de situação de moradia na rua pode ser um importante marcador de vulnerabilidade em várias áreas da vida desses indivíduos. Aqueles que estiveram em situação de rua, em algum momento da vida, apresentaram os piores escores de gravidade nas áreas álcool, problemas médicos, psiquiátricos, emprego e suporte familiar, além de mais sintomas depressivos, prisão por roubo e renda insuficiente para pagar necessidades básicas. 
Regressão de Poisson múltipla para situação de rua de usuários de crack em tratamento em Centros de Atenção Psicossocial para Álcool e Drogas (CAPSad) de seis capitais brasileiras.

\begin{tabular}{|c|c|c|c|}
\hline & $\mathbf{R P}$ & Valor de $p$ & IC95\% \\
\hline Intercepto & 0,269 & $<0,001$ & 0,$196 ; 0,369$ \\
\hline Internado por problemas psicológicos/psiquiátricos & 1,220 & 0,072 & 0,$983 ; 1,516$ \\
\hline Sintomas depressivos & 1,501 & 0,002 & 1,$163 ; 1,939$ \\
\hline Ideação suicida & 1,135 & 0,199 & 0,$936 ; 1,376$ \\
\hline Preso por porte de drogas & 1,154 & 0,129 & 0,$959 ; 1,389$ \\
\hline Preso por roubo & 1,315 & 0,002 & 1,$103 ; 1,567$ \\
\hline Fisicamente agredido/abusado por alguém que conhecia & 1,032 & 0,743 & 0,$854 ; 1,247$ \\
\hline Agredido/Abusado sexualmente & 1,108 & 0,347 & 0,$894 ; 1,374$ \\
\hline Vítima de um crime violento & 1,113 & 0,246 & 0,$929 ; 1,333$ \\
\hline Não possui renda suficiente para pagar necessidades básicas & 1,359 & 0,004 & 1,$359 ; 1,676$ \\
\hline Gênero masculino & 1,057 & 0,632 & 0,$842 ; 1,327$ \\
\hline
\end{tabular}

IC95\%: intervalo de 95\% de confiança; RP: razão de prevalência.

Nota: para essa análise, foram excluídos os sujeitos que possuíam alguma questão não preenchida, totalizando um $n$ final de 550 sujeitos. Em negrito valores de $p$ significativos $(p<0,05)$.

O estudo sobre determinantes e condicionantes da saúde traz uma importante contribuição para a compreensão da complexidade dos aspectos que envolvem a dependência química e sua interface com padrões socioeconômicos e demográficos. Dentro da perspectiva relacional de fatores sociais, o nível socioeconômico, entendido conceitualmente por nível educacional, renda e trabalho, influencia a saúde e padrões de uso de usuários de drogas 19,20. Nesse sentido, os resultados encontrados neste trabalho são consistentes com a literatura, que aponta que a população de usuários de crack brasileira é composta predominantemente por homens adultos jovens, solteiros, de baixa escolaridade, desempregados e de baixa renda 2,21,22. Dados nacionais apontam que 35,5\% da população em situação de rua do país referem o uso abusivo de álcool ou de outras drogas como a principal motivação para passar a viver e morar na rua, e $28 \%$ informam já ter passado por alguma casa ou clínica de recuperação para dependentes químicos 23. Estudos internacionais com populações em situação de rua apontam que esses indivíduos identificam o uso de substâncias como uma das principais razões para sua perda de moradia e permanência na rua 24 . Da mesma forma, pessoas que dependem de programas de moradia, ou que não têm moradia estável, apresentam maior índice de abuso e/ou dependência de substâncias ilícitas, o que pode levar ao envolvimento com a justiça criminal e gerar relações tensas com familiares e amigos, afetando, ainda mais, sua capacidade de garantir a habitação 25 .

Ao avaliarmos o status de moradia como um importante marcador de vulnerabilidade social, diferentes aspectos são considerados. No presente trabalho, os fatores sociodemográficos, especificamente no que diz respeito ao gênero, os sujeitos do estudo eram predominantemente homens. Entretanto, verifica-se, na literatura, um número significativo de mulheres usuárias de crack em situação de rua. Tais resultados são semelhantes a estudos prévios, que mostram uma tendência de aumento na prevalência de mulheres usuárias de crack. Ressalta-se, também, que as questões de gênero permeiam a discussão a respeito do acesso a tratamento por mulheres. Estudos afirmam que o estigma, a autoimagem e o papel social desempenhado e esperado na sociedade pela mulher, além do padrão de uso de drogas, podem estar associados às diferenças na procura por tratamento nesta população 26,27.

Em relação a outros fatores sociodemográficos, os resultados mostraram semelhanças em todos os níveis de escolaridade, sendo que 12,1\% do total eram analfabetos. Esses dados confirmam os indicadores de educação brasileiros, que apontam para um alto índice de crianças e jovens fora da escola, ingresso tardio no sistema educacional, baixo aproveitamento/desempenho escolar e defasagem idade/série 28,29. 
Fatores adicionais que compõem os critérios de vulnerabilidade social como status socioeconômico, status de moradia e situação legal também foram investigados. Nesse sentido, é importante ressaltar que a população estudada apresentou porcentuais elevados de indivíduos com renda de até um salário mínimo (37,2\%). Não foi encontrada diferença significativa entre os dois grupos em relação à renda, entretanto, quando testados no modelo de regressão multivariada, a variável "possuir renda suficiente para pagar necessidades básicas" apresentou números inferiores para os sujeitos em situação de rua. Esses dados conduzem à discussão a respeito de trabalho e emprego dos usuários de crack. Dados nacionais demonstram que os usuários de crack estão mais propensos a se engajarem em atividades de trabalho esporádico ou autônomo (65\%), sexo em troca de dinheiro (7,46\%) e atividades ilícitas como roubo e tráfico de drogas $(6,4 \%)$ como forma de obtenção de dinheiro 2 . A inserção como mão de obra do tráfico de drogas aparece como uma estratégia de sobrevivência frente à vulnerabilidade econômica, deixando essa população exposta à violência das organizações criminosas 30 .

$\mathrm{Na}$ amostra estudada, os usuários apresentam grande envolvimento com questões legais, registrando que o uso de crack tem uma intrínseca relação com a violência. Estudos sobre o perfil de usuários de crack demonstram que eles estão mais expostos a ambientes violentos, a envolvimento com crimes e a aumento de fatores de risco para a sua saúde 1,31. Nesse sentido, tais indivíduos têm uma maior probabilidade de detenção e envolvimento com o crime ${ }^{21}$. Da mesma forma, a urgência pelo uso da droga (craving) e a falta de condições financeiras para sustentar o consumo contribuem para que o usuário de crack se engaje em atividades ilícitas, tais como roubos e assaltos, a fim de custear o uso desta substância ${ }^{32}$. Os resultados apresentados sugerem que existem diferenças entre as categorias de avaliação de gravidade de dependência do ASI-6 nos dois grupos, muito embora com pouca variação em algumas áreas.

Chamam atenção, entretanto, alguns aspectos relacionados à saúde física e mental dos usuários. A literatura aponta que, em diferentes países, existe uma associação importante entre moradores de rua e o uso de substâncias ilícitas, bem como com doenças como HIV, hepatites e tuberculose 33 . Neste estudo, a presença de HIV e hepatite foi significativamente maior entre os usuários de crack moradores de rua. Considerando que a rota de transmissão é semelhante entre esses tipos de vírus (sexual, perinatal e contaminação sanguínea), entende-se que isso está de acordo com os dados encontrados que relatam maiores crimes de violência e abuso sexual, deixando o indivíduo mais vulnerável à contaminação 13,34. Isso caracteriza uma população com maior probabilidade de apresentar diminuição da imunidade, menor acesso a ações de prevenção, bem como uma maior probabilidade de contato com outros enfermos, visto que muitos dormem em albergues ou locais inapropriados e a contaminação por tuberculose, por exemplo, torna-se facilitada 35. Outro dado relevante é a maior prevalência de sintomas depressivos encontrada nos usuários de crack moradores de rua, somada a um maior risco de cometer suicídio. Esse achado está de acordo com um estudo prévio que aponta uma importante prevalência de uso de cocaína e comorbidades psiquiátricas, em especial sintomas depressivos 31 .

Em especial, indivíduos em situação de rua apresentam maior vulnerabilidade social 36, têm menos suporte social e pouco acesso a serviços e ações de prevenção e apoio social. Esse grupo tem uma maior probabilidade de não ter suas necessidades atendidas e, desta forma, estratégias de enfrentamento e acesso a serviços para esta população em especial devem estar entre as prioridades das políticas públicas 10,23.

De acordo com Siqueira 37, a "desvalorização da dignidade humana”, associada a problemas crônicos, tais como fome, miséria, insalubridade e desemprego, contribuem para que ocorra o crescimento da violência em diferentes níveis da sociedade. Países periféricos como o Brasil carecem da oferta de direitos sociais elementares, gerando condições de miséria, pobreza extrema, falta de acesso à escolaridade e saúde, não sendo possível dissociar o comportamento individual do contexto em que ocorre 37 . Nesse mesmo sentido, Bastos 38 refere que, no Brasil, há forte presença da chamada violência estrutural, com uma pronunciada associação entre a violência urbana e o envolvimento com o tráfico de drogas. Alerta, ainda, que perpetradores e vítimas de violência têm perfil semelhante no país: jovens, pobres e oriundos de comunidades de periferia. Estudos etnográficos mostram que a simples presença do morador de rua provoca mal-estar na população que circula pelos espaços urbanos. O morador de rua é associado ao fracasso, à transgressão, a comportamentos violentos, a roubos e à sujeira. O uso de drogas é visto como uma condição intrínseca para suportar a situação de rua e, ao mesmo tempo, como um ato imoral e de falta de atenção ao próprio corpo 39,40 . 
Muitos usuários destacam que as sucessivas perdas e fracassos servem como motivação para o ingresso na situação de rua e que o uso de crack aumenta a sensação de exclusão ${ }^{41}$. No ambiente da rua, a presença do tráfico e o consumo de substâncias ganham mais relevância em função de sua visibilidade em uma cena aberta 2,32 .

Ao se discutir os resultados do presente estudo, identifica-se que o grau de exclusão social de usuários de crack que, em algum momento, estiveram expostos à situação de falta de moradia e gravidade do uso, deixa-os ainda mais vulneráveis a possíveis situações de violência. Assim, torna-se necessário resgatar o compromisso ético do Estado no papel protetivo às pessoas em situação de vulnerabilidade, com base na proposição de ações que contribuam para a qualidade de vida desses sujeitos e que levem em conta contextos socioculturais específicos 42 . Políticas públicas, serviços e programas devem estar adequados a uma estratégia de acolhimento integral, com profissionais preparados para o atendimento das demandas no sistema de saúde.

\section{Limitações do estudo}

Os resultados do presente estudo devem ser vistos com cautela na medida em que o método de amostragem foi por conveniência e não probabilístico, sendo a amostra selecionada em centros de atendimento e destinada a indivíduos que buscaram tratamento em serviços públicos especializados. Muito embora exista uma possível variabilidade quanto à definição do status de moradia na rua, o grupo de usuários de crack já pertence a uma situação de extrema vulnerabilidade social, em que possivelmente esta condição é um marcador relevante. Além disso, por se tratar de uma análise de dados secundários, é possível que existam limitações quanto ao poder estatístico das análises, uma vez que o cálculo amostral foi baseado no desfecho previsto para o estudo principal. Entretanto, por ser multicêntrico e com bom tamanho amostral, o estudo contempla uma amostra de diferentes regiões brasileiras, contribuindo para a compreensão mais ampla desse fenômeno em nosso país.

\section{Conclusão}

No Brasil, ainda são escassos os estudos multicêntricos, em especial aqueles voltados à população em situação de rua. Os resultados do presente trabalho possibilitaram conhecer de forma mais abrangente a gravidade do uso de drogas e situação de moradia de usuários de crack em tratamento em CAPSad de diferentes regiões do Brasil. Nos últimos anos temos observado a implantação e ampliação de serviços e dispositivos voltados aos usuários de drogas dentro de modelos que preconizam a integralidade e a humanização do cuidado, como por exemplo, abordagens de rua, programas de redução de danos e terapia comunitária. Entende-se que tal população encontra-se em situação de vulnerabilidade social e que abordagens projetadas de acordo com o perfil e as necessidades específicas poderão subsidiar políticas públicas e ações voltadas para um cuidado mais qualificado, tanto na área da saúde como na da assistência social. 


\section{Colaboradores}

S. C. Halpern participou da concepção, análise e interpretação dos dados do projeto e redação do artigo. J. N. Scherer e V. Roglio contribuíram na análise e interpretação dos dados e revisão crítica do artigo. C. Dalbosco e A. Sordi colaboraram na revisão crítica do conteúdo intelectual do artigo. F. Ornell colaborou na redação do artigo. S. Faller e F. Pechansky participaram da concepção do projeto e revisão crítica do conteúdo do artigo. F. Kessler e L. von Diemen contribuíram com revisão crítica do conteúdo do artigo e aprovação da versão final. Os autores são responsáveis por todos os aspectos do trabalho na garantia da exatidão e integridade de qualquer parte do artigo.

\section{Agradecimentos}

À Secretaria Nacional de Políticas sobre Drogas (SENAD), Ministério da Justiça.

\section{Referências}

1. Carvalho HB, Seibel SD. Crack cocaine use and its relationship with violence and HIV. Clinics 2009; 64:857-66.

2. Bastos FI, Bertoni N. Pesquisa nacional sobre o uso de crack: quem são os usuários de crack e/ou similares do Brasil? Quantos são nas capitais brasileiras? Rio de Janeiro: Instituto de Comunicação e Informação Científica e Tecnológica em Saúde, Fundação Oswaldo Cruz; 2014.

3. Santos Cruz M, Andrade T, Bastos FI, Leal E, Bertoni N, Villar LM, et al. Key drug use, health and socio-economic characteristics of young crack users in two Brazilian cities. Int J Drug Policy 2013; 24:432-8.
4. Laranjeira R. II Levantamento Nacional de Álcool e Drogas (LENAD) - 2012. São Paulo: Instituto Nacional de Ciência e Tecnologia para Políticas Públicas de Álcool e Outras Drogas/Universidade Federal de São Paulo; 2014

5. Siliquini R, Morra A, Versino E, Renga G. Recreational drug consumers: who seeks treatment? Eur J Public Health 2005; 15:580-6.

6. Santos Cruz M, Andrade T, Bastos FI, Leal E, Bertoni N, Lipman L, et al. Patterns, determinants and barriers of health and social service utilization among young urban crack users in Brazil. BMC Health Serv Res 2013; 13:536. 
7. Faller S, Peuker AC, Sordi A, Stolf A, SouzaFormigoni ML, Cruz MS, et al. Who seeks public treatment for substance abuse in Brazil? Results of a multicenter study involving four Brazilian state capitals. Trends Psychiatry Psychother 2014; 36:193-202.

8. Watts MJ, Bohle HJ, May M, Action P. The space of vulnerability: the causal structure of hunger. Prog Hum Geogr 1993; 17:291-305.

9. Mulia N, Zemore SE. Social adversity, stress, and alcohol problems: are racial/ethnic minorities and the poor more vulnerable? J Stud Alcohol Drugs 2012; 73:570-80.

10. Argintaru N, Chambers C, Gogosis E, Farrell S, Palepu A, Klodawsky F, et al. A crosssectional observational study of unmet health needs among homeless and vulnerably housed adults in three Canadian cities. BMC Public Health 2013; 13:577.

11. Inciardi JA, Surratt HL, Pechansky F, Kessler F, von Diemen L, Silva EM, et al. Changing patterns of cocaine use and HIV risks in the south of Brazil. J Psychoact Drugs 2006; 38:305-10.

12. Roy É, Robert M, Fournier L, Laverdière E, Berbiche D, Boivin J-F. Predictors of residential stability among homeless young adults: a cohort study. BMC Public Health 2016; 16:131.

13. Grangeiro A, Holcman MM, Onaga ET, Alencar HD, Placco AL, Teixeira PR. Prevalence and vulnerability of homeless people to HIV infection in São Paulo, Brazil. Rev Saúde Pública 2012; 46:674-84.

14. Faller S. Resultados acerca do uso de substâncias psicoativas no Brasil a partir de estudos multicêntricos [Tese de Doutorado]. Porto Alegre: Faculdade de Medicina, Universidade Federal do Rio Grande do Sul; 2015.

15. American Psychiatric Association. Diagnostic and statistical manual of mental disorders. 4th Ed. Arlington: American Psychiatric Association; 2000
16. McLellan AT, Cacciola JC, Alterman AI, Rikoon SH, Carise D. The Addiction Severity Index at 25: origins, contributions and transitions. Am J Addict 2006; 15:113-24.

17. Kessler F, Cacciola J, Alterman A, Faller S, Souza-Formigoni ML, Cruz MS, et al. Psychometric properties of the sixth version of the Addiction Severity Index (ASI-6) in Brazil. Rev Bras Psiquiatr 2012; 34:24-33.

18. Montgomery DC, Peck EA, Vining GG. Introduction to linear regression analysis. 3rd Ed. New York: Wiley; 2006.

19. Galea S, Vlahov D. Social determinants and the health of drug users: socioeconomic status, homelessness, and incarceration. Public Health Rep 2002; 117 Suppl:S135-45.

20. Victora CG, Barreto ML, do Carmo Leal M, Monteiro CA, Schmidt MI, Paim J, et al. Health conditions and health-policy innovations in Brazil: the way forward. Lancet 2011; 377:2042-53.

21. Ferreira Filho OF, Turchi MD, Laranjeira R, Castelo A. Epidemiological profile of cocaine users on treatment in psychiatrics hospitals, Brazil. Rev Saúde Pública 2003; 37:751-9.

22. Horta RL, Horta BL, Rosset AP, Horta CL. Perfil dos usuários de crack que buscam atendimento em Centros de Atenção Psicossocial. Cad Saúde Pública 2011; 27:2263-70.

23. Ministério da Saúde. Saúde da população em situação de rua: um direito humano. Brasília: Ministério da Saúde; 2014.

24. Collins SE, Malone DK, Clifasefi SL. Housing retention in single-site housing first for chronically homeless individuals with severe alcohol problems. Am J Public Health 2013; 103 Suppl 2: S269-74. 
25. Gabrielian S, Bromley E, Hellemann GS, Kern RS, Goldenson NI, Danley ME, et al. Factors affecting exits from homelessness among persons with serious mental illness and substance use disorders. J Clin Psychiatry 2015; 76:e469-76.

26. Peixoto C, Prado CHO, Rodrigues CP, Cheda JND, Mota LBT, Veras AB. Impact of clinical and socio-demographic profiles in treatment adherence of patients attending a day hospital for alcohol and drug abuse. J Bras Psiquiatr 2010; 59:317-21.

27. Mulia N, Schmidt L, Bond J, Jacobs L, Korcha R. Stress, social support and problem drinking among women in poverty. Addiction 2008; 103:1283-93.

28. Ministério da Educação. Educação brasileira: indicadores e desafios: documentos de consulta. Brasília: Ministério da Educação; 2013.

29. Ministério da Educação. Censo Escolar da Educação Básica 2016: notas estatísticas. Brasília: Ministério da Educação; 2017.

30. Zaluar A. Sociability in crime. Culture, form of life or ethos? Vibrant Virtual Brazilian Anthropology 2014; 11:12-46.

31. Kessler FHP, Terra MB, Faller S, Stolf AR, Peuker CA, Benzano D, et al. Crack users show high rates of antisocial personality disorder, engagement in illegal activities and other psychosocial problems. Am J Addict 2012; 21:370-80.

32. Toledo L, Góngora A, Bastos FIPM. À margem: uso de crack, desvio, criminalização e exclusão social - uma revisão narrativa. Ciênc Saúde Coletiva 2017; 22:31-42.

33. Leiva-Hidalgo J, Madrigal-Méndez A, SalasSegura D. Seroprevalencia de hepatitis B, C y VIH en indigentes en Costa Rica. Reva Costarric Salud Pública 2013; 22:113-8.

34. Thomas DL, Astemborski J, Rai RM, Anania FA, Schaeffer M, Galai N, et al. The natural history of hepatitis $\mathrm{C}$ virus infection: host, viral, and environmental factors. JAMA 2000; 284:450-6
35. García C. Tuberculosis en grupos de riesgo en la Región Metropolitana. 2008. Rev Chil Enferm Respir 2010; 26:105-11.

36. Ministério da Saúde. Manual sobre o cuidado à saúde junto à população em situação de rua. Brasília: Ministério da Saúde; 2012.

37. Siqueira JE. Ensaio sobre vulnerabilidade humana. In: Zoboli ELCP, Barchifontaine CP, organizadores. Bioética, vulnerabilidade e saúde. Aparecida: Ideias \& Letras; 2007. p. 13-28.

38. Bastos FI. Structural violence in the context of drug policy and initiatives aiming to reduce drug-related harm in contemporary Brazil: a review. Subst Use Misuse 2012; 47:1603-10.

39. Rui T. Nas tramas do crack: etnografia da abjeção. São Paulo: Terceiro Nome; 2014.

40. Medeiros RP. Bêbados, noiados e moradores de rua. In: Fernandez OFRL, Andrade MM, Nery Filho A, organizadores. Drogas e políticas públicas: educação, saúde coletiva e direitos humanos. Salvador: EDUFBA/Brasília: ABRAMD; 2015. p. 19-40.

41. Adorno RCF, Rui T, Silva SL, Malvasi PA, Vasconcellos MP, Gomes BR, et al. Etnografia da cracolândia: notas sobre uma pesquisa em território urbano. Saúde Transform Soc 2013; 4:4-13.

42. Nery Filho A, Lorenzo C, Diz F. Usos e u. Prevenção dos problemas relacionados ao uso de drogas: capacitação para conselheiros e lideranças comunitárias. Brasília: Secretaria $\mathrm{Na}-$ cional de Políticas sobre Drogas, Ministério da Justiça/Florianópolis: Núcleo Multiprojetos de Tecnologia Educacional, Universidade Federal de Santa Catarina; 2014. p. 127-41. 


\section{Abstract}

The study had the goal to evaluate psychoactive substance use severity, violence, physical and emotional health of crack users who seeks specialized treatment in Psychosocial Care Centers for Alcohol and Drugs (CAPSad) concerning housing status. This is a multicenter cross-sectional study in six Brazilian capitals with 564 crack users categorized into two groups (1) users who have been homeless sometime in life $(n=266)$ and $(2)$ individuals who have never lived on streets $(n=298)$. To assess the substance use severity and the characteristics of the individuals, the Addiction Severity Index, $6^{\text {th }}$ version (ASI-6) was used. Group 1 users showed worse indicators regarding alcohol, medical and psychiatric problems, employment and family support subscales, as well as greater involvement with legal problems, violence, sexual abuse, suicide risk and health related problems such as HIV/AIDS, hepatitis and tuberculosis. In addition they have lower income to pay for basic needs. After analysis and control for possible confounders, not having enough income to pay for basic needs, showing depression symptoms, and having been arrested for theft remained statistically significant. This study evaluated more deeply drug use severity and housing status of crack users. Interventions developed in outpatient treatment should be designed and tailored to specific profiles and demands of drug users, especially homeless individuals.

Substance-Related Disorders; Crack; Homeless Persons; Health Vulnerability;

Social Vulnerability

\section{Resumen}

El objetivo de este estudio fue evaluar la gravedad del uso de sustancias psicoactivas, situaciones de violencia, salud fisica y emocional de consumidores de crack que buscan atención en Centros de Atención Psicosocial para Alcohol y Drogas (CAPSad), en relación con el status de acceso a la vivienda. Se trata de un estudio multicéntrico en seis capitales brasileñas, con 564 consumidores de crack categorizados en dos grupos: (1) consumidores sin techo $(n=266)$ y (2) quienes nunca fueron $\sin$ techo $(n=$ 298). Para evaluar la gravedad del uso de sustancias y las características de los individuos se utilizó el Addiction Severity Index, 6a versión (ASI-6). Los consumidores del grupo 1 demostraron peores indicadores, en relación a las subescalas alcohol, problemas médicos, psiquiátricos, trabajo y apoyo familiar, además de una mayor implicación en problemas legales, violencia, abuso sexual, riesgo de suicidio y problemas de salud como VIH/SIDA, hepatitis y tuberculosis, además de contar con menos renta para pagar necesidades básicas. Tras los análisis multivariados ajustados para posibles confusores, no contar con renta suficiente para pagar necesidades básicas, presentar sintomas depresivos y haber sido preso por robo fueron significativos. Este trabajo evaluó de forma más amplia la gravedad del consumo de drogas y la situación de acceso a la vivienda de consumidores de crack. Se deben proyectar enfoques desarrollados en los dispositivos de tratamiento extra-hospitalarios, adecuados según perfiles y demandas especificas de los consumidores, en especial aquellos en situación de desamparo.

Trastornos Relacionados con Sustancias; Crack; Personas sin Hogar; Vulnerabilidad en Salud; Vulnerabilidad Social
Recebido em 04/Mar/2017

Versão final reapresentada em 09/Mai/2017

Aprovado em 06/Jun/2017 